\title{
COMMENT
}

Check for updates

\section{Decoding bat immunity: the need for a coordinated research approach}

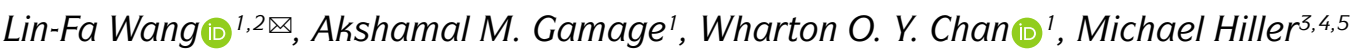 \\ and Emma C. Teeling 6
}

\begin{abstract}
Understanding antiviral immune responses in bats, which are reservoirs for many emerging viruses, could aid the response to future epidemics. Here, we discuss five key areas in which greater consensus among the bat research community is necessary to drive breakthroughs in the field.
\end{abstract}

With more than 1,400 species that collectively account for $\sim 20 \%$ of all living mammals, bats serve as a rich resource for understanding mammalian evolution and adaptation $^{1}$. Bats belong to the Chiroptera order and are the only mammals capable of self-powered flight. They are also remarkably diverse in terms of their physiology, social biology, sensory perception, ecological habitats and diet. In recent years, bats have garnered attention as suspected reservoirs for viruses that can cause disease in humans, including the SARS-related coronaviruses ${ }^{2}$. This observation has been attributed to a tolerant (that is, non-pathological) bat immune response to these viruses $^{3}$, as well as to the high species diversity within the chiropteran order ${ }^{4}$. Recent studies have further supported the idea of a uniquely adapted immune system in bats, which limits inflammatory responses upon infectious challenge ${ }^{5}$. In addition to providing a better understanding of viral disease tolerance mechanisms, studying bat immunity can also contribute to the areas of comparative mammalian immunology, ageing and longevity, and immunometabolism. However, research into bat immunity suffers from multiple hurdles, including the scarcity of bat-specific research tools and a lack of standardized model systems. In this Comment article, we summarize five major technical competencies that will be vital for improving our understanding of bat immunology. These are the development of structural and functional genomics, immortalized bat cell lines, primary cell culture and organoids, a single-cell transcriptomics atlas and captive breeding colonies (FIG. 1). We highlight the key areas where greater coordination and consensus amongst the bat research community is needed to better allocate resources and drive breakthroughs in this nascent space.

Research into the chiropteran immune system gathered momentum in the early 2010 s, spurred on by observations of a hitherto unrecognized diversity of filoviruses and coronaviruses in various bat species, and a major advance was the sequencing of the first bat genomes in 2013 (REF. ${ }^{6}$ ). Genome sequences are currently available for approximately 50 bat species, and on-going efforts by the Bat $1 \mathrm{~K}$ consortium to generate reference-quality genomes for all living bat species ${ }^{7}$ will provide a much needed foundational resource for the subsequent development of immunological tools. The availability of bat-derived cell lines has also enabled in vitro experimentation and hypothesis testing ${ }^{8}$. Transcriptomic and molecular studies using cell lines have provided novel insights into how bat cells respond to infection. However, despite being an accessible and tractable resource, bat cell lines have several limitations, including the lack of complex cell interactions that are present in vivo and artefacts imposed by the process of immortalization. The use of primary tissue (including total leukocytes from peripheral blood, splenocytes and bone marrow-derived myeloid cell populations) and bat-derived organoids can overcome some of these limitations ${ }^{9}$. Primary immune cell populations, such as bone marrow-derived macrophages, enable comparative studies with their specific human or mouse cell counterparts. Compared with cell lines, organoids better recapitulate the spatial arrangement and cellular heterogeneity seen in tissues such as the airways and intestine. A limitation here would be the difficulty to procure primary material, unless there is access to a captive breeding colony. Nevertheless, resources for the above three technical competencies are at least partially available at present and provide researchers with a sufficient toolkit to initiate in vitro investigations into the bat immune response.

Progressing beyond these in vitro systems, there is a need to focus on systems-wide approaches to study the bat immune response in vivo to both infectious and non-infectious challenges. Antibody panels for multiplexed flow-cytometry or mass-cytometry are difficult to establish as bat-specific antibody probes are rarely available. While cross-reactive antibodies that bind to conserved markers across mammalian species have been used to delineate some immune cell populations in bats, the high investments required for developing 


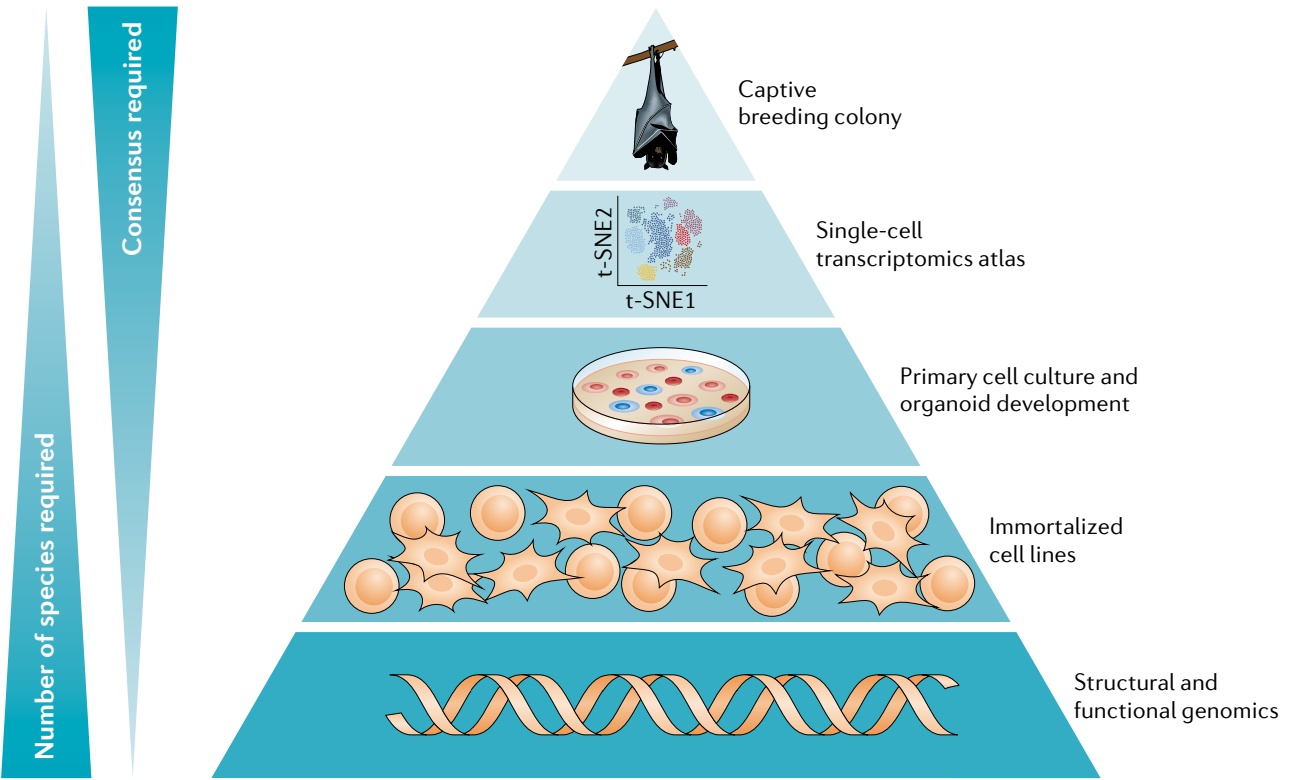

b

\begin{tabular}{|llllll|}
\hline Species & Suborder & Diet & Distribution & $\begin{array}{l}\text { Example } \\
\text { viruses hosted }\end{array}$ & $\begin{array}{l}\text { Captive } \\
\text { colony? }\end{array}$ \\
\hline $\begin{array}{l}\text { Eonycteris spelaea } \\
\text { (Cave nectar bat) }\end{array}$ & Yinpterochiroptera & Nectar & $\begin{array}{l}\text { Southeast Asia, } \\
\text { China, India }\end{array}$ & Reoviruses & Yes \\
$\begin{array}{l}\text { Rousettus aegyptiacus } \\
\text { (Egyptian fruit bat) }\end{array}$ & Yinpterochiroptera & Fruit & $\begin{array}{l}\text { Sub-Saharan Africa, } \\
\text { Egypt }\end{array}$ & Filoviruses & Yes \\
\hline $\begin{array}{l}\text { Pteropus spp. } \\
\text { (Megabats) }\end{array}$ & Yinpterochiroptera & Fruit & $\begin{array}{l}\text { Southeast Asia, } \\
\text { Australia, Madagascar }\end{array}$ & Henipaviruses & Yes \\
\hline $\begin{array}{l}\text { Rhinolophus sinicus } \\
\text { (Chinese rufous } \\
\text { horseshoe bat) }\end{array}$ & Yinpterochiroptera & Insects & $\begin{array}{l}\text { China, Vietnam, } \\
\text { Nepal, India }\end{array}$ & Coronaviruses & No \\
$\begin{array}{l}\text { Artibeus jamaicensis } \\
\text { (Jamaican fruit bat) }\end{array}$ & Yangochiroptera & Fruit & $\begin{array}{l}\text { Central and South } \\
\text { America }\end{array}$ & Arenaviruses & Yes \\
\hline
\end{tabular}

Fig. 1 | Key areas for consensus in bat immunology. a | Key technical competencies necessary to advance understanding of bat immunity. At the top of the pyramid are competencies that will only be feasible to establish for a limited number of species and for which the importance of consensus increases. b | Five major bat species proposed for captive breeding colony establishment and in vivo model development.

bat species-specific antibodies make it unlikely that antibody-based studies will serve as a primary tool for the unbiased interrogation of bat immune systems, in contrast to their central role in human and mouse immunology research. Instead, we propose using singlecell RNA sequencing (scRNA-seq) to generate cellular atlases of the bat immune system. This approach will enable the characterization of bat immune cell populations and their responses to stimulation at a high resolution, and can eventually be scaled up to analyse representative species from all major bat genera. This goal is within the immediate horizon, given the maturity of bioinformatics pipelines and the increasing ease and cost-effectiveness of generating scRNA-seq datasets. Such data would provide unprecedented insights into the diversity and similarities of immune system structure and function within the chiropteran order. However, significant logistical obstacles exist in achieving this goal, including reaching a consensus on key representative species to be analysed. Overcoming these challenges would likely benefit from having a similar operational approach to that of the Bat $1 \mathrm{~K}$ project, by having local collaborators and ultimately sequencing centres across multiple countries.

The establishment of captive breeding colonies for in vivo bat research comprises the final and most advanced competency (FIG. 1 a). Although important early findings have been derived from bats caught in the wild, this model is neither sustainable nor safe. Given the difficulties in establishing a bat colony for a given species, and the significant effort in generating the downstream species-specific competencies listed above, we believe a discussion within the community is crucial in order to reach a consensus on the key bat species that should be used to establish in vivo models. We propose an initial list of species from five bat genera that we believe are representative of the evolutionary and geographical diversity seen in bats; Rousettus aegyptiacus, Rhinolophus sinicus, Pteropus spp. and Eonycteris spelaea from the Yinpterochiroptera suborder and Artibeus jamaicensis 
from the Yangochiroptera suborder. This list is based on practical criteria including research interest derived from naturally hosted viral diversity, size, conservation status and species for which prior evidence of established colonies exist (FIG. 1 b). It should be noted that difficulties in maintaining insectivorous bats in captivity will likely skew the selected model systems towards frugivorous species.

Taken together, this work will fundamentally improve our understanding of the bat immune system. Ultimately, a better appreciation of how bats respond to viruses will inform the development of immunomodulatory strategies for treating patients with adherent inflammation, including during severe viral infections. The renewal of interest in the field of bat immunology that has arisen from the COVID-19 pandemic should provide an impetus to re-strategize and coordinate efforts to take full advantage of these fascinating and important animal models.
1. Teeling, E. C. et al. Bat biology, genomes, and the Bat $1 \mathrm{~K}$ project: to generate chromosome-level genomes for all living bat species. Annu. Rev. Anim. Biosci. 6, 23-46 (2018).

2. Letko, M., Seifert, S. N., Olival, K. J., Plowright, R. K. \& Munster, V. J. Bat-borne virus diversity, spillover and emergence. Nat. Rev. Microbiol. 18, 461-471 (2020).

3. Irving, A. T., Ahn, M., Goh, G., Anderson, D. E. \& Wang, L.-F. Lessons from the host defences of bats, a unique viral reservoir. Nature $\mathbf{5 8 9}$, 363-370 (2021).

4. Mollentze, N. \& Streicker, D. G. Viral zoonotic risk is homogenous among taxonomic orders of mammalian and avian reservoir hosts. Proc. Natl Acad. Sci. USA 117, 9423-9430 (2020).

5. Ahn, M. et al. Dampened NLRP3-mediated inflammation in bats and implications for a special viral reservoir host. Nat. Microbiol. 4 , 789-799 (2019).

6. Zhang, G. et al. Comparative analysis of bat genomes provides insight into the evolution of flight and immunity. Science 339 456-460 (2013)

7. Jebb, D. et al. Six reference-quality genomes reveal evolution of bat adaptations. Nature 583, 578-584 (2020).

8. Banerjee, A., Misra, V., Schountz, T. \& Baker, M. L. Tools to study pathogen-host interactions in bats. Virus Res. 248, 5-12 (2018).

9. Zhou, J. et al. Infection of bat and human intestinal organoids by SARS-CoV-2. Nat. Med. 26, 1077-1083 (2020).

\section{Competing interests}

The authors declare no competing interests. 